\title{
Transplante pulmonar como tratamento de pacientes com fibrose cística
}

\section{Lung transplantation in patients with cystic fibrosis}

\author{
Karina Turaça ${ }^{1}$, Paulo Manuel Pêgo Fernandes ${ }^{2}$, \\ Marcos Naoyuki Samano ${ }^{3}$, Alexandre Kazantzi ${ }^{4}$
}

Turaça K, Fernandes PMP, Samano MN, Kazantzi A. Transplante pulmonar como tratamento de pacientes com fibrose cística. / Lung transplantation in patients with cystic fibrosis. Rev Med (São Paulo). 2013 jan.-mar.;92(1):69-74.

RESUMO: Introdução. A partir de 1980 com o uso da ciclosporina e novas técnicas operatórias o transplante pulmonar se tornou uma terapia bem estabelecida para pacientes portadores de doenças crônicas em estágio avançado. Atualmente o procedimento é realizado em mais de 150 centros em todo mundo. A sobrevida global estabelecida para receptores de transplante de pulmão é cerca de cinco anos, podendo variar de acordo com os fatores de risco do paciente. Apesar do aumento da qualidade de vida ser bem estabelecido, a indicação de transplante deve ser cuidadosamente realizada, pois há um risco inerente de mortalidade precoce (aproximadamente $10 \%$ em 1 ano). A fibrose cística, uma doença genética autossômica recessiva, é a terceira maior causa de indicação de transplante pulmonar, uma vez que a grande maioria dos pacientes portadores dessa patologia evolui a óbito por insuficiência respiratória, devido a muitos anos de infecção pulmonar crônica. Objetivos. O objetivo desse estudo é descrever a evolução e avaliar a sobrevida de pacientes submetidos a transplante de pulmão. Metodologia. Realizou-se um estudo retrospectivo com revisão de prontuários de 30 pacientes transplantados por fibrose cística entre maio de 2004 e dezembro de 2010. Foram analisados os seguintes parâmetros: sexo, idade, peso, estatura, tipo sanguíneo, associação com comorbidades, tempo de doença, função cardíaca, avaliação pré-operatória com teste de caminhada e espirometria. No doador, foi foram descritos, sexo, idade, IMC, gasometria, tempo de intubação e leucograma. No transplante foi avaliado o tempo operatório, tempo de UTI e de internação. Resultados. A média de idade dos pacientes foi de 27,4+9,22 anos, sendo 2 na faixa pediátrica (abaixo de 18 anos). Foram 14 pacientes do sexo masculino e 16 do sexo feminino. O IMC médio foi de $19,1+3,0 \mathrm{Kg} / \mathrm{m}^{2}$ e todos os pacientes faziam acompanhamento nutricional. O tempo médio de doença foi de 20,8 + 9,7 anos e o tempo médio que os pacientes permaneceram na lista de transplante foi de $20,99+8,83$ meses. Todos os pacientes realizaram espirometria pré-transplante, a média dos resultados obtidos foram: VEF1(L) 3,8+12,3; VEF1(\%) 24,22+9,2; CVF(L) 7,29+25,10; CVF(\%) 39,41+18,09; VEF1/ CVF(L) 2,42+9,7; VEF1/CVF(\%) 23,04+25,14. A taxa de sobrevida foi de $72,3 \%$ em cinco anos com queda acentuada no primeiro ano e estabilização após esse período. Após o transplante a média do resultado das espirometrias realizadas 6 meses após o transplante foi de: VEF1(L) 2,44+0,66; VEF1(\%) 73,20+18,13; CVF(L) 2,66+0,71; CVF(\%) 68,2+15,74; VEF1/ CVF(L) 4,13+1,77; VEF1/CVF(\%) 96,66+43,91. Discussão. A faixa etária predominante dos 30 pacientes estudados corresponde a pacientes mais jovens. Sete pacientes evoluíram a óbito no período estudado, levando a uma sobrevida média de $72,3 \%$ em cinco anos, menor do que a descrita na literatura. Os demais pacientes apresentaram melhora na qualidade de vida em médio e longo prazo. Conclusão. O transplante pulmonar para pacientes portadores de fibrose cística em é hoje uma terapia bem estabelecida por apresentar uma melhora significativa da qualidade de vida. Com o desenvolvimento de novas técnicas operatórias e novos medicamentos imunossupressores a sobrevida aumentou substancialmente, apesar de ainda manter-se elevada no primeiro ano após o transplante.

DESCRITORES: Transplante pulmonar; Fibrose cística; Sobrevida.

$1^{\circ}$ lugar Painel Área Cirúrgica - XXXI Congresso Médico Universitário da FMUSP - COMU 2012.

1. Acadêmica do $3^{\circ}$ ano do Curso de Medicina da Faculdade de Medicina da Universidade de São Paulo. e-mail: karina_kt@hotmail. com.

2. Professor Livre-Docente, Departamento de Cardiopneumologia, Disciplina de Cirurgia Torácica, Faculdade de Medicina da Universidade de São Paulo, Instituto do Coração da FMUSP

3. Professor Doutor, Departamento de Cardiopneumologia, Disciplina de Cirurgia Torácica da Faculdade de Medicina da Universidade de São Paulo (FMUSP.

4. Aluno de complementação especializada do Departamento de Cardiopneumologia do Instituto do Coração da Faculdade de Medicina da Universidade de São Paulo.

Endereço para correspondência: Marcos N. Samano. Av. Dr. Enéas de Carvalho Aguiar, 44 - 2ªndar - Bloco II - Sala 9. São Paulo, SP. CEP: 05403-000. e-mail: marcos.samano@incor.usp.br 


\section{INTRODUÇÃO}

A fibrose cística é uma doença genética autossômica recessiva com mutação no gene CTFR (cystic fibfrosis transmembrane conductor regulator).

A Fibrose Cística é a terceira maior causa de indicação de transplante pulmonar no mundo, uma vez que a grande maioria dos pacientes portadores dessa patologia evoluem para deterioração do quadro respiratório, devido a muitos anos de infecção pulmonar.

Atualmente o transplante pulmonar é realizado em mais de 150 centros em todo mundo, seu estabelecimento se deu a partir de 1980 com uso de medicações imunossupressoras e novas técnicas operatórias.

A sobrevida global estabelecida para receptores de transplante de pulmão é cerca de cinco anos, podendo variar de acordo com os fatores de risco do paciente.

\section{OBJETIVOS}

O objetivo desse estudo é descrever a evolução e avaliar a sobrevida de pacientes portadores de Fibrose Cística submetidos a transplante de pulmão no Instituto do Coração HC-FMUSP.

\section{METODOLOGIA}

Realizou-se um estudo retrospectivo com revisão de prontuários de 30 pacientes transplantados por fibrose cística entre abril de 2004 e dezembro de 2010.

Foram analisados os seguintes parâmetros: sexo, idade, peso, estatura, associação com comorbidades, tempo de doença, função cardíaca e avaliação pré e pós-operatória com espirometria. No doador, foi foram descritos: sexo, idade, IMC, gasometria, tempo de intubação e prevalência de tabagismo. No transplante foi avaliado o tempo operatório, tempo de isquemia, tempo de UTI, ventilação mecânica e intubação.

\section{RESULTADOS}

De abril de 2004 até dezembro de 2010, foram transplantados 30 pacientes com fibrose cística na instituição. Foram 14 pacientes do sexo feminino (46,7\%) e 16 do sexo masculino $(53,3 \%)$. Dados referentes a casuística (Tabela 1), comorbidades (Tabela 2) e função pulmonar (Gráficos 1 e 2), doadores (Tabela 3) e dados referentes a cirurgia (Tabela 4 ).

Tabela 1. Dados referentes à casuística

\begin{tabular}{|c|c|}
\hline \multicolumn{2}{|c|}{ CASUÍSTICA } \\
\hline DADOS & RESULTADOS \\
\hline Média de Idade & $27,4 \pm 9,22$ anos ( 2 abaixo de 18 anos) \\
\hline IMC médio & $19,1 \pm 3,0 \mathrm{Kg} / \mathrm{m}^{2}$ \\
\hline Tempo médio de doença & $20,8 \pm 9,7$ anos \\
\hline Tempo médio de espera em lista & $20,99 \pm 8,83$ meses \\
\hline
\end{tabular}

Tabela 2. Dados referentes à comorbidades

\begin{tabular}{lc}
\hline \multicolumn{1}{c}{ COMORBIDADES } \\
\hline \multicolumn{2}{c}{ DADOS } \\
\hline Prevalência de disfunção pancreática exócrina & RESULTADOS \\
Prevalência de Diabetes Mellitus & $60 \%(18 / 30)$ \\
Pacientes colonizados por P. aeruginosa & $20 \%(6 / 30)$ \\
Pacientes colonizados por B. cepacia & $83 \%(25 / 30)$ \\
\hline
\end{tabular}

Tabela 3. Dados referentes a doadores

\begin{tabular}{ll}
\hline \multicolumn{1}{c}{ DADOS } & \multicolumn{1}{c}{ DOADORES } \\
\hline Sexo & \multicolumn{1}{c}{ RESULTADOS } \\
Média de Idade & Feminino: $11(36,7 \%)$ - Masculino: $19(63,3 \%)$ \\
Gasometria Média & de $25,1 \pm 10,9$ anos \\
Prevalência de tabagismo & $376,2 \pm 67,4 \mathrm{pO} 2 / \mathrm{FiO} 2 \mathrm{mmHg}$ \\
Tempo médio de intubação & $6,7 \%(2 / 30)$ \\
\hline
\end{tabular}


Gráficos 1 e 2. Dados referentes a função pulmonar
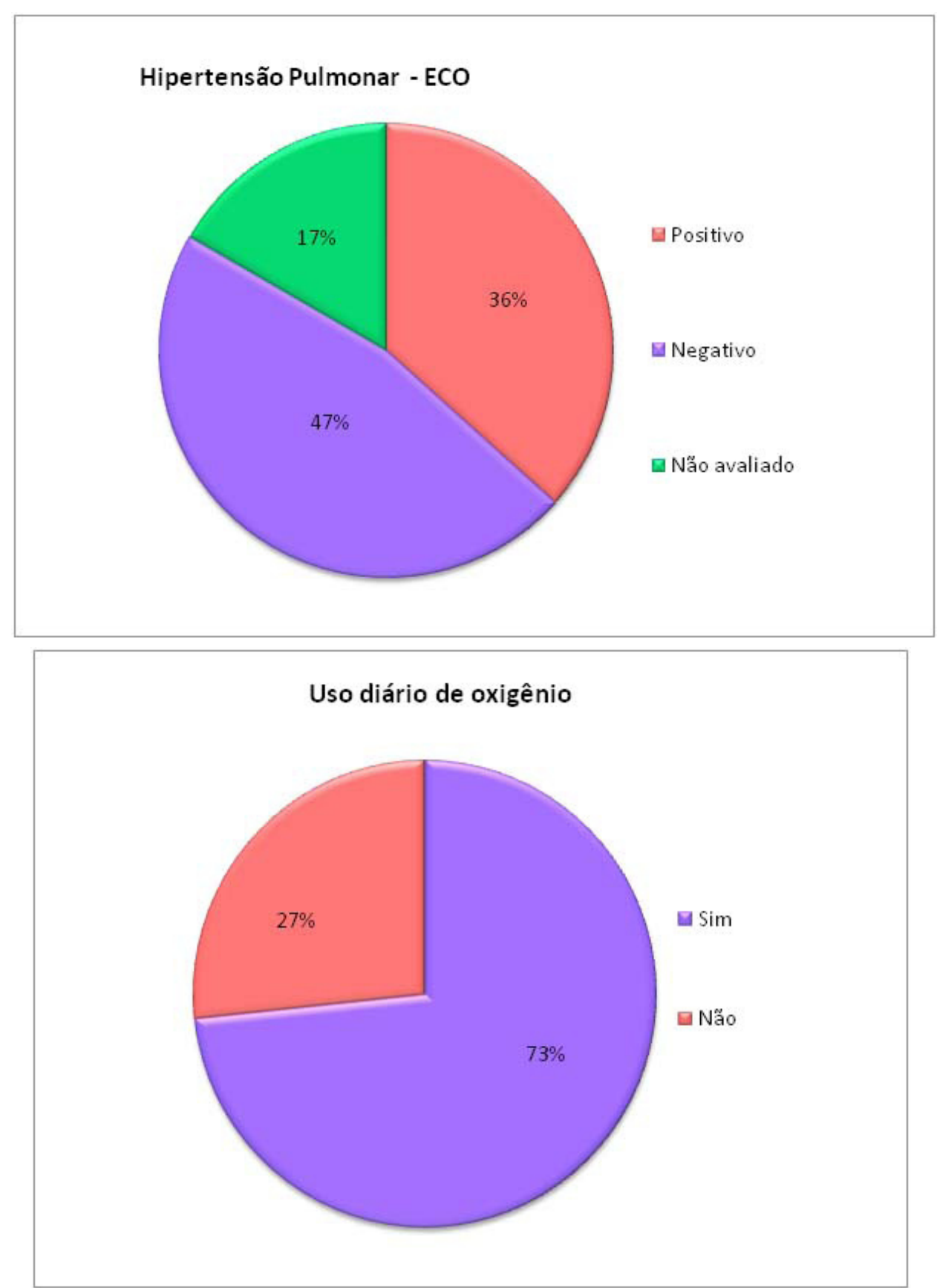

Tabela 3. Dados referentes a doadores

\begin{tabular}{ll}
\multicolumn{1}{c}{ DADOS } & \multicolumn{1}{c}{ DOADORES } \\
\hline Sexo & \multicolumn{1}{c}{ RESULTADOS } \\
\hline Média de Idade & Feminino: $11(36,7 \%)$ - Masculino: $19(63,3 \%)$ \\
Gasometria Média & de $25,1 \pm 10,9$ anos \\
Prevalência de tabagismo & $376,2 \pm 67,4 \mathrm{pO} 2 / \mathrm{FiO} 2 \mathrm{mmHg}$ \\
Tempo médio de intubação & $6,7 \%(2 / 30)$ \\
\hline
\end{tabular}


Turaça $\mathrm{K}$ et al. Transplante pulmonar como tratamento de pacientes com fibrose cística.

Tabela 4. Dados referentes à cirurgia

\begin{tabular}{ll}
\hline & CIRURGIA \\
\hline DADOS & RESULTADOS \\
\hline Média de tempo de isquemia & $1^{\circ}$ lado: $284 \pm 78,4$ min $-2^{\circ}$ lado: $472 \pm 98,3$ min \\
Tempo médio de cirurgia & $714,7 \pm 96,9$ minutos \\
\hline Tempo médio de permanência em UTI & $9,9 \pm 6,3$ dias \\
Tempo médio de ventilação mecânica & $37,2 \pm 41,09$ horas \\
\hline
\end{tabular}

A sobrevida e a evolução dos pacientes estão relatadas nos Gráficos 3 e 4, esta última foi avaliada por meio da realização de espirometrias ao longo dos anos. A sobrevida encontrada foi de $92 \%$ em um ano e $77 \%$ em cinco anos.

Gráficos 3 e 4. Gráficos de sobrevida e evolução dos pacientes

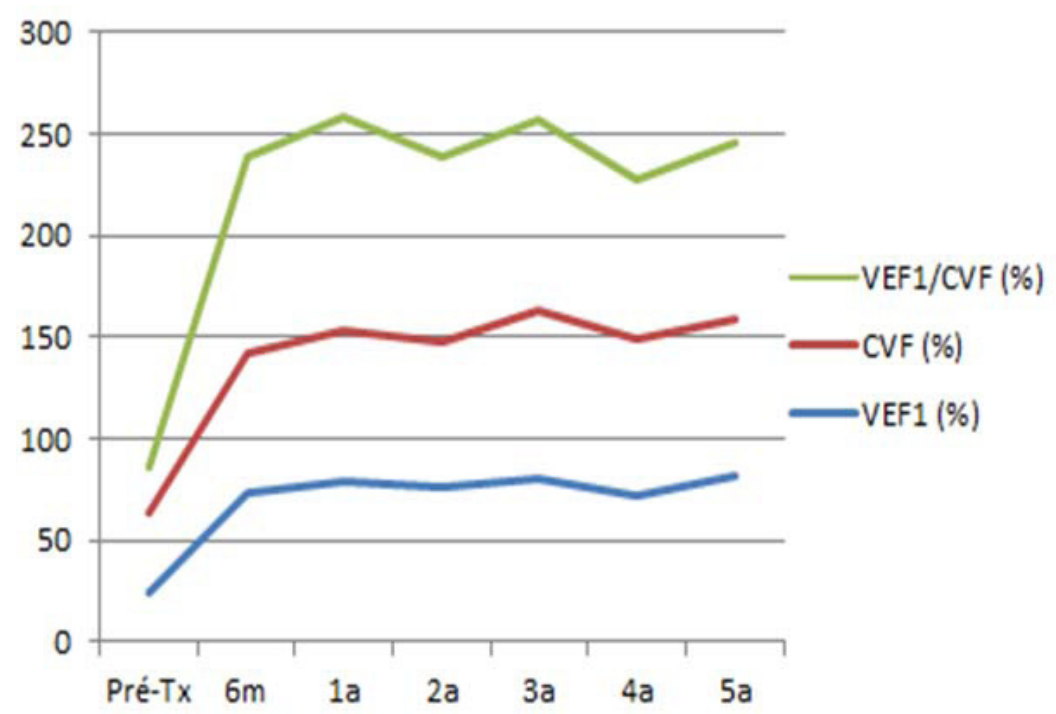

Survival Functions

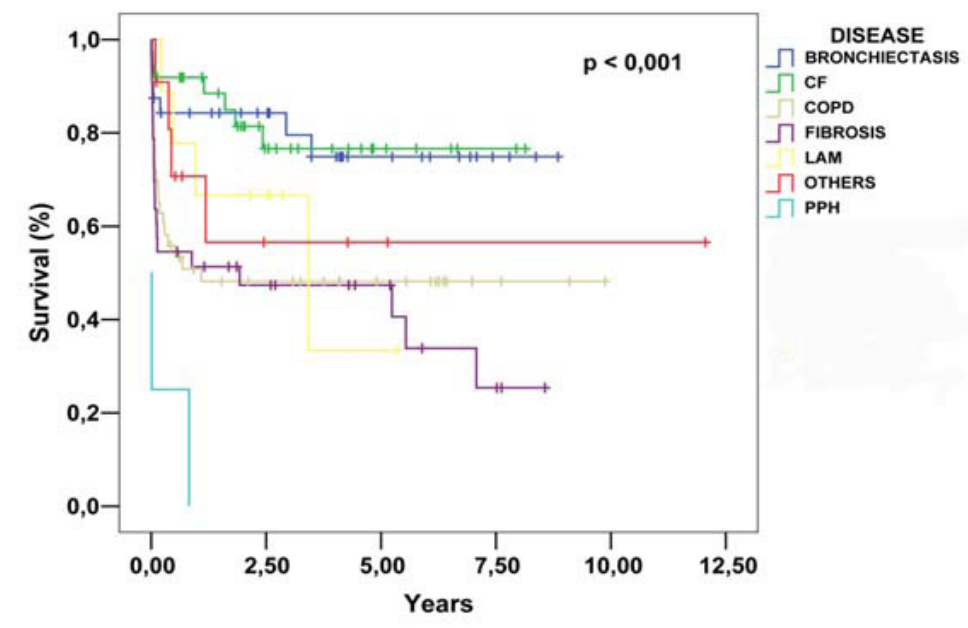




\section{DISCUSSÃO}

A casuística é composta predominantemente por indivíduos jovens e com baixo IMC, apesar de todos os pacientes terem realizado acompanhamento nutricional. A função pulmonar dos pacientes apresentava uma deterioração, tendo em vista que $73 \%$ deles eram oxigênio-dependentes.

O tempo prolongado em lista aguardando transplante $(20,9 \pm 8,8$ meses $)$ decorre da legislação brasileira de priorizar a data de inclusão em lista e não a gravidade da doença.

A incidência de disfunção primária do enxerto foi de apenas $10 \%$ na casuística estudada. Nenhum paciente morreu devido a septicemia após o procedimento, a mortalidade aguda foi relacionada à insuficiência hepática grave, tromboembolismo pulmonar e derrame cerebral.

Apesar de maior mortalidade em 30 dias, em comparação com outras séries de relatos mundiais, o grupo apresentou a segunda maior taxa de sobrevida em um ano (92\%) e em cinco anos (77\%).

A experiência adquirida pelo grupo de Transplante Pulmonar do Instituto do Coração do HCFMUSP, com a realização de 30 transplantes pulmonares bilaterais em um período de 7 anos é a maior experiência brasileira .

\section{Tabela 5}

\begin{tabular}{lcccccccccc}
\hline & $\begin{array}{c}\text { Current } \\
\text { series }\end{array}$ & $\begin{array}{c}\text { Inci } \\
\text { et al. } \\
(2012)\end{array}$ & $\begin{array}{c}\text { Meachery et } \\
\text { al. }(2008)\end{array}$ & $\begin{array}{c}\text { Bech } \\
\text { et al. } \\
(2004)\end{array}$ & $\begin{array}{c}\text { Algar } \\
\text { et al. } \\
(2008)\end{array}$ & $\begin{array}{c}\text { Quattrucci } \\
\text { et al. } \\
(2003)\end{array}$ & $\begin{array}{c}\text { Wiebe } \\
\text { et al. } \\
(1998)\end{array}$ & $\begin{array}{c}\text { Spahr } \\
\text { et al. } \\
(2007)\end{array}$ & $\begin{array}{c}\text { Egan } \\
\text { et al. } \\
(2002)\end{array}$ & $\begin{array}{c}\text { ISHLT } \\
(2011)\end{array}$ \\
\hline $\mathrm{n}$ & 30 & 100 & 176 & 29 & 78 & 55 & 35 & 57 & 123 & 4828 \\
$\begin{array}{l}1 \text { ano } \\
\text { (sobrevida) }\end{array}$ & $92 \%$ & $88 \%$ & $82 \%$ & $89 \%$ & $75 \%$ & $79 \%$ & $91 \%$ & $95 \%$ & $81 \%$ & $82.6 \%$ \\
$\begin{array}{l}5 \text { anos } \\
\text { (sobrevida) }\end{array}$ & $77 \%$ & $72 \%$ & $62 \%$ & $80 \%$ & $58 \%$ & $58 \%$ & $76 \%$ & $67 \%$ & $59 \%$ & $58.6 \%$ \\
$\begin{array}{l}30 \text { dias } \\
\text { (mortalidade) }\end{array}$ & $10 \%$ & $5 \%$ & NR & $3.5 \%$ & $19.2 \%$ & $17.8 \%$ & NR & $3.5 \%$ & $8.1 \%$ & $6 \%$ \\
\hline
\end{tabular}

\section{CONCLUSÃO}

Apesar do número reduzido de pacientes, o trabalho apresenta uma taxa de sobrevida em longo prazo comparável a outros relatos mundiais. A fibrose cística é a segunda maior causa de indicação de transplante pulmonar em nosso serviço e apresenta a melhor sobrevida quando comparada com as demais comorbidades.

\section{REFERÊNCIAS}

1. Christie JD, Edwards LB, Kucheryavaya AY, Benden C, Dobbels F, Kirk R, Rahmel AO, Stehlik J, Hertz MI. The Registry of the International Society for Heart and Lung Transplantation: Twenty-eighth adult lung and heartlung transplant report - 2011. J Heart Lung Transplant. 2011;30(10):1104-22. doi: 10.1016/j.healun.2011.08.004.

2. Yankaskas JR, Mallory GB. Lung transplantation in cystic fibrosis: consensus conference statement. Chest. 1998;113(1):217-26. doi:10.1378/chest.113.1.217

3. Adler FR, Aurora P, Barker DH, et al. Lung transplantation for cystic fibrosis. Proc Am Thorac Soc. 2009;6 (8):619-33. doi: 10.1513/pats.2009008-088TL.

4. Okay TS, Oliveira WP, Raiz-Júnior R, Rodrigues JC, Del Negro GM. Frequency of the deltaF508 mutation in 108 cystic fibrosis patients in Sao Paulo: comparison with reported Brazilian data. Clinics (Sao Paulo). 2005;60(2):131-4. http:// dx.doi.org/10.1590/S1807-59322005000200009

5. Park M, Vieira Costa EL, Pontes Azevedo LC, et al. Extracorporeal membrane oxygenation as a bridge to

pulmonary transplantation in Brazil: are we ready to embark upon this new age? Clinics 2011;66(9):1659-61. doi: $10.1590 / \mathrm{S} 1807-59322011000900027$

6. Samano MN, Minamoto H, Junqueira JJM, et al. Bronchial Complications following lung transplantation. Transplant Proc. 2009;41(3):921-6. doi: 10.1016/j. transproceed.2009.01.047.

7. Inci I, Stanimirov O, Benden C, et al. Lung transplantation for cystic fibrosis: a single center experience of 100 consecutive cases. Eur J Cardiothorac Surg. 2012;41(2):435-40. doi: 10.1016/j.ejcts.2011.06.017.

8. Meachery G, De Soyza A, Nicholson A, et al. Outcomes of lung transplantation for cystic fibrosis in a large UK cohort. Thorax. 2008;63(8):725-31. doi: 10.1136/ thx.2007.092056.

9. Bech B, Pressler T, Iversen M, et al. Long-term outcome of lung transplantation for cystic fibrosis -Danish results. Eur J Cardiothorac Surg 2004;26(6):1180-6. doi: 10.1016/j. ejcts.2004.08.015. 
Turaça $\mathrm{K}$ et al. Transplante pulmonar como tratamento de pacientes com fibrose cística.

10. Algar FJ, Cano JR, Moreno P, et al. Results of lung transplantation in patients with cystic fibrosis. Transplant Proc. 2008;40(9):3085-7. doi: 10.1016/j. transproceed.2008.08.120.

11. Quattrucci S, Cimino G, Bertasi S, et al. Lung transplantation for cystic fibrosis in Italy. Transplant Proc. 2008;40(6):2003-5. doi: 10.1016/j.transproceed.2008.05.036.

12. Wiebe K, Wahlers T, Harringer W, vd Hardt H, Fabel H, Haverich A. Lung transplantation for cystic fibrosis - a single center experience over 8 years. Eur J Cardiothorac Surg. 1998;14(2):191-6. doi: 10.1016/S1010-7940(98)00163-8.

13. Spahr JE, Love RB, Francois M, Radford K, Meyer KC. Lung transplantation for cystic fibrosis: current concepts and one center's experience. J Cyst Fibros. 2007;6(5):334-50. http:// dx.doi.org/10.1016/j.jcf.2006.12.010.

14. Egan TM, Detterbeck FC, Mill MR, et al. Long term results of lung transplantation for cystic fibrosis. Eur J Cardiothorac Surg. 2002;22(4):602-9. doi: 10.1016/S1010-7940(02)00376-7. 